\title{
Tabletop Crisis Training and the Requirements for Computer-Based Simulators
}

\author{
John Dowell \\ Ergonomics and HCI Unit, Department of Psychology \\ University College London, 26 Bedford.Way \\ London WC.IH OAP, UK \\ Phone +44 (0)171 3877050 \\ E-mailj.dowell@ucl.ac.uk
}

Synopsis

Catastrophic accidents and natural disasters require many professionals to work together if the emergency relief operation is to be effective. Coordination between agents is especially difficult when different agencies are involved and when they must contend with severe uncertainties, hazards and time limitations. Coordination, then, is an important area of training need. The emergency agencies have wellestablished training practices and rely, in particular, on tabletop exercises. In a tabletop exercise, a physical model and script of a crisis scenario are used for learning 'by discussion' about how to manage the emergency operation. Although tabletop exercises are widely practised, it may not be assumed that they best satisfy the training needs of coordination. This paper first identifies an agenda of such training needs. An account is then given of a tabletop exercise and by considering this exercise with regard to the training needs agenda, requirements for computer-based simulations are proposed. The paper concludes with a summary assessment of ICCARUS, a computer-based simulator for training fire service officers. As a state-of-the art system, ICCARUS indicates the extent to which the requirements for coordination training are currently met by computer-based simulators. 


\section{Keywords}

emergency management; coordination; tabletop training; training simulators

\section{Introduction}

The emergency and rescue operation which follows a catastrophic accident or natural disaster must invariably contend with severe uncertainties and hazards, and with limited time. The fire at the Kings Cross underground station was just such an occasion: in unfamiliar territory, without a reliable and shared assessment of the hazards, and with poor communications, the Fire Brigade and Police had to decide which would be a safe evacuation route before the 'flashover' into the booking hall that eventually claimed many lives (Fennel, 1988). Because each occasion of a catastrophe brings its own uncertainties and hazards (Lagadec, 1990), contingency plans cannot be reduced to mere procedure; rather, problems must be recognised as they emerge and resources deployed to effect a best solution.

The difficulties of managing an emergency operation increase significantly with the involvement of different agencies and their 'agents'. In addition to the emergency services, major emergency operations can require the participation of local authorities, specialist expert groups and commercial organisations. Consequently, there will be complex inter-dependencies between the activities of different agents; for example, ambulance crews leaving the scene of a catastrophe will expect instructions from the police who themselves will expect to be continuously notified by the hospitals of their currently available capacity. This is the problem of coordination.

The degree of coordination achieved in the management of an emergency operation will have a direct bearing on the operation's effectiveness. Failures in coordination were a notable concern expressed in the public enquiry report on the Kings Cross fire: "The court was left with the impression that there had been a breakdown of communications at command level between the emergency services. 
Each diligently pursued its own duty but there was a lack of liaison between them" (Fennel, 1988). However, any analysis of coordination is likely to be possible from a number of different perspectives. One perspective which has shown promise in analysing coordination in other collective decision-making tasks is provided by the framework of 'distributed cognition' (Hutchins, 1990; Cicourel, 1990). Distributed cognition describes perception, planning, action, communication and knowledge as phenomena distributed between each member of a group addressing a common set of tasks and goals (loosely, a metaphor of distributed computation). This framework assumes (see Rasmussen, Brehmer and Leplat,1991) that the social organisation of cognitive activity is more significant than individual cognition in accounting for the performance of collective work.

Coordination Issues in Emergency Management: An Agenda of Training Needs

The framework of distributed cognition has been used (Dowell, 1995) to identify general cognitive issues of coordination visible in the emergency operation following the Clapham railway accident. Those issues are now briefly summarised here, and distinguished as issues of planning, action, communication, and knowledge.

\section{Coordination Issues of Planning}

Each agency participating in an emergency operation will have contingency plans drawn up in readiness for the different kinds of catastrophe that may occur; those plans will be known to each agent. Yet because catastrophes are fundamentally unpredictable, contingency plans must be partial, high level, and conditional; accordingly, for the occasion of each catastrophe, contingency plans must be interpreted by each agency and each agent, regardless of level of authority. If this plan interpretation is conducted implicitly and informally, coordination will clearly suffer. In particular, the necessary variance between the priorities of different agencies and of different agents may become dislocated, leading to priorities which are duplicated, in conflict, or 
which neglect important concerns. Planning is also conducted under "high temporal constraints" (Samurçay and Rogalski, 1993) and it must be 'reactive' to domain events. Both of these factors force monolithic, decompositional planning to give way to a more opportunistic but fragmented form of planning.

Coordination requires that contingency plans are explicitly interpreted, and that conflicts can be recognised and resolved. It requires that the priorities of all agents, whilst necessarily varying, should conform with a unitary goal structure for the emergency operation. It also requires opportunistic planning to be made explicit.

\section{Coordination issues of plan implementation (action)}

Since plans cannot be reduced to simple procedures, the sequencing of actions of different agents during the emergency operation is determined by mutual constraints established between those agents; it does not proceed from individual contingency plans. In this way, individuals are said to create "behavioural dependencies" (Hutchins, 1990) between each other. Action becomes reactive to the effects of other agents' actions, in other words, the emergency operation " emerges in the interaction between actions, it does not follow some master plan" (Hutchins 1991).

Coordination relies on the recognition and negotiation of behavioural dependencies, and it requires that the actions of each agent, and their effects, are visible to those who will need to act on them.

\section{Coordination issues of communication}

An essential feature of communication during emergency operations is that authoritative, validated and accurate information disseminates progressively but often only slowly. Information channels will invariably be limiting, commonly making communications asynchronous (Borodzicz, Aragones and Pidgeon, 1993) and even disallowing responses. 
Coordination requires authoritative, validated and accurate information to be disseminated as rapidly as possible. It relies on the timely 'passing on' of information to those who may need it. In the absence of feedback, coordination requires an understanding of how others will respond to messages and actions.

\section{Coordination issues of roles and task knowledge}

If coordination requires each agent to know how others will respond to their messages and actions, then it is apparent that different agents must have knowledge of each others tasks. There must then, be at least a partial redundancy in knowledge and skills (Emery and Trist, 1973). This redundancy of task knowledge is said to be a pre-requisite for modifying the real divisions of labour (Hutchins, 1990), whether through violation or negotiation.

Coordination requires agencies and their agents to have redundant knowledge and skills. It requires differences in the division of labour to be negotiated. The cognitive horizon (Hutchins, 1990) which delimits the uniqueness and redundancy of an agent's knowledge is critical to recognising which agents need extra support: if coordination means giving extra support where it is needed, then cognitive horizons must be sufficiently broad.

More generally, even when individual responses are well trained, ineffectiveness can result if agencies and their agents are not well coordinated. Coordination has been identified as a major factor in the overall effectiveness of emergency operations. For example, the potential consequences (Dowell, 1995) of poor coordination in the aftermath of the Clapham railway accident included another train colliding with the wreckage, the electrocution of rescuers, and delays in evacuating passengers and attending to the injured.

The vital importance of coordination was concluded by the public inquiry following the Kings Cross fire. The inquiry saw training as the principal way of improving coordination: "There ought to be joint exercises between the emergency services because I am satisfied that if 
such joint exercises had taken place, communications would have been better and some of the problems which presented themselves would not have proved as difficult as they did on that night" (Fennel, 1988). These recommendations for training were echoed in the public inquiry report (Hidden, 1989) following the Clapham railway accident. The Hidden report specifically identified tabletop exercises as the way to provide training in coordination. The following section describes such a tabletop exercise.

The cognitive issues of coordination discussed in this section might be considered as an agenda of training needs for emergency management. By considering the tabletop exercise against this agenda, general user requirements for computer-based training simulators for the emergency management task are later formulated.

\section{The Tabletop Exercise: Training in Emergency Management}

Training practices are well established within the emergency agencies, preparing personnel in the roles they will play through each stage of an emergency operation - from protection and rescue to containment and restoration. Training is currently provided in three, mutually supportive ways. First, classroom-based tutoring provides trainees with the principles of good emergency management and exposes them to their agency's contingency plans for different classes of catastrophe. Second, 'live' training exercises are conducted annually to provide learning 'by doing' in as near realistic conditions as possible. An example of a recent 'live' exercise in the UK adopted a catastrophe scenario in which an aircraft had collided with a train. For the day-long exercise, a train was purposely wrecked, professional actors were employed as victims, and a full scale emergency operation was staged involving all agents from all relevant agencies.

The third way in which emergency responses are trained is through tabletop simulation exercises. Tabletop exercises employ a physical, scale model of a catastrophe to promote discussion about the 
organisation and implementation of the emergency operation. The trainees may include agents from each of the agencies that would be involved in the real emergency operation. A variant of the tabletop exercise is the 'paper-feed' exercise which uses the passing of text by hand to simulate the time-course of events during an emergency and the responses of the trainees to those events.

Tabletop exercises are a vital form of training for the emergency agencies (Overy, 1993). In contrast with classroom tuition, they provide experience in the collective management of emergencies without the costs associated with live exercises. The following is an account of such a tabletop exercise observed by the author in 1993. The account will be used in the subsequent section to consider requirements for computer-based simulations for emergency management training.

Participating in the tabletop exercise were some thirty middle-level managers from the agencies that would typically be called upon in the case of a serious incident in the area of London where the exercise was held. They included personnel from the three main emergency services, hospital managers, local authority managers - and area and operations managers from British Rail. The exercise was run by a 'Moderator' and his assistant from the training branch of a major rescue agency. Introducing the participants to the exercise, the Moderator explained that: "the recognised need for a more professional response to emergencies [...] must encompass a multi-agency approach." $\mathrm{He}$ emphasised that: " team work is of the essence [...] and no agency can work in isolation. Teamwork is vital for the success of the operation." The Moderator was even more specific about the nature and timing of this need for communication: "early in the disaster, there must be good, medium level interfacing between different agencies."

The exercise was directed towards a physical, scale model representing an elevated railway line and adjoining urban areas. The model represented some 800 metres of empty track, at one end of which was a small station. Buildings and other features were given by 
miniatures and roads were painted. The Moderator asked the participants to view the model as representing a named local station with which all were familiar. Since the model neither faithfully nor accurately represented this area, the Moderator summarised key features of the scenario, such as schools and churches, and went so far as to enumerate their occupants. The participants were then invited to examine the model for themselves. Circulating freely around the model, the participants could interrogate the Moderator about details of the scenario, for example, the direction from which the station would normally be accessed.

The Moderator divided the participants into four 'syndicates', each syndicate having two members from each agency. This done, the Moderator began the exercise by delivering the following script:

"It's Tuesday the 8th of June, 8:20 am. A call is received from Mrs Jones at St Michael's church hall by the station. She says: "There's been a bang on the railway and I can hear people shouting. I'm going to investigate." This call is received by the three major services. I want you to imagine that you're the controller from your service. Within each of your syndicate groups, please consider the following questions:

a) who would you notify?

b) what would your service response be to that message in terms of personnel?

c) how long would it take you to get there?

d) for the railway participants, how would you be aware of the incident, who would be sent and how would they get there?"

Once these questions were delivered, participants withdrew into their syndicates to discuss their answers. After fifteen minutes, the Moderator re-convened the exercise into one group for a debriefing of answers. In this way, four answers were elicited to each of the original questions. The answers, their differences, and their implications for inter-agency coordination were discussed, a discussion led by the Moderator. 
The emergency scenario was now extended. The Moderator's assistant arranged on the tabletop model, miniatures representing a collision between an eight coach commuter train and a tanker train. Representatives of the fire, police and ambulance services were then invited to place on the scenario, models of their respective units; for example a fireman placed two model fire engines near to St Michael's church. At the same time, a short video was shown of a train colliding with a solid obstruction: the Moderator asked participants to appreciate the destructive forces released by the momentum of a train.

The second question given to the participants was as follows: "What are the priorities of the first persons on the scene from each agency?" The participants again withdrew into their syndicates to discuss their answers, and after fifteen minutes, were re-convened for a debriefing of the second question. The following is a partial transcript of this debriefing:

Policeman1: "Our first priority is to scale the incident, and to identify the residual hazards and the casualties. We would contact Scotland Yard and declare it a major incident. We would likely then evacuate those two schools. ....... We want to immediately set up a rendezvous point for emergency vehicles ..... Within the first 1 to 30 minutes we would close roads. We would ask for a hospital designation and then consider access to the incident for ambulances coming from those hospitals. We would set up a 1way system for ambulances coming in and out, and we'd have a total exclusion of all other traffic. We'll set up types of injury to type of ambulance and hospital resources. We would also consider getting the electricity shut off."

Fireman1: "Our first priority is to get the line shut down, to protect people coming off the train and the services going in. By this time, you'd probably have shocked and confused people streaming all over the track. We'd also lay out hoses in case of fire" 
Moderator: "There is no fire at the moment. Any other responses from the London fire brigade, please? "

Fireman2: "We're all round the wrong side of the train at the moment. We need to be on the other side - 'cause access will be easier up the grassy bank on that other side."

Ambulanceman1: "There could be 750 injured people in those 8 carriages so I'd have called a major incident by now. We would also get up a rendezvous and a control point on the other side (of the train)."

Policeman2: "Hopefully the staff at the station would be aware by now that there is a major incident. They would almost certainly have heard the accident, given that it's happened only a few hundred yards from the station and so they should already be aware of what's happened and have stopped the trains to prevent any further disaster."

Railwayman1: "Well our first person on the scene is of course the driver; and assuming he's $\mathrm{OK} . . . ? "$

Moderator: "- He's dead ... and there's no guard"

Railwayman1: "Ah ... he's dead ... and there's no guard, ... right, so it's not a dangerous goods train, then. So its down to the driver, then, of the tanker train, to inform the signal box and then to put detonators on the track. mmh"

Fireman 1: "But how will we know if the electricity is off? We'll be frazzled if we're not told!"

Railwayman2: "Well it's our duty to get the lines switched off - to block other trains."

Moderator: "So the priority has to be to stop the trains, then get the power off? Is this the overriding priority?"

Railwayman2: "Yes - but in a crash like this, the current would almost certainly be off already ... because of short circuits caused by the wreckage." 
The tabletop exercise continued in this way; the scenario was extended (the tanker train begins leaking a toxic and volatile liquid), and five further questions were posed by the Moderator. The exercise was closed after the debriefing of the final question.

\section{Requirements for Simulations to Train Coordination}

Tabletop exercises are a principal form of training in emergency management provided by the UK emergency agencies, and were recommended by the public inquiries into the Kings Cross fire and the Clapham railway accident. As we have seen, both those inquiries identified coordination as a particular area of training need. Yet it might not be assumed that tabletop exercises offer the best, or most complete training in coordination. Earlier (Section 1), an agenda of training needs, focused on the issues of coordination, was cited. In this section, the agenda of coordination training needs is used to review the instance of a tabletop exercise, described above. The review discusses the extent to which the tabletop exercise appeared to satisfy the agenda of training needs. On the basis of this review are derived general requirements for computer-based simulators for training coordination.

\section{Assessment of the tabletop training exercise}

It was apparent that the participants came to the exercise trained in the roles and responsibilities of their own agencies. Fireman1, for instance, knew he must request the track isolation before proceeding, and Railwayman2 knew the procedures for responding to requests for isolation; the exercise enabled both to recognise and discuss the coordination of track isolation. In this way, tabletop exercises confront the coordination issue that in spite of each agent making the right decisions and taking the right actions, ineffectiveness will result if those agents are poorly coordinated. Tabletop exercises respond to the need for collective training to complement individual training. 
Training coordinated planning. The basis of the individual training that preceded the tabletop exercise was the contingency plans of the respective agencies. Yet contingency plans were not consulted during the tabletop exercise, nor were they explicitly mentioned. Rather, they appeared implicitly in the participants' responses. For instance, Policeman3 interpreted the plan for halting other railway traffic as requiring the station staff to inform the signalbox. Railwaymanl interpreted the plan as putting the responsibility on the driver of the commuter train. In fact the driver was a fatal victim of the accident, and so the tanker driver should have taken on the responsibility. It would appear that tabletop exercises provide training in the collective interpretation of contingency plans necessary for coordination. Nevertheless, because the process of interpretation remains tacit, it may fail to make apparent when plans are mis-interpreted - or when they are ignored altogether.

Conflicting views about priorities were disclosed in the interpretation of contingency plans. Policemanl, for instance, did not identify as a priority the removal of the traction current; instead, he considered the evacuation of the schools and the setting up of a road traffic system. It was apparent in the discussion that the other participants identified the electrical isolation as a common priority. So, through discussion, the tabletop exercise helped all the participants to recognise common priorities in managing the emergency operation. In this way, tabletop exercises provide training in resolving conflicts between different agents' and agencies' priorities that may often often produce coordination difficulties.

Although the tabletop exercise followed a 'storyboard' sequence of events, for instance - the initial crash and the subsequent leakage of flammable liquid, it did not have a temporal dimension. Events and the responses to those events were not constrained by any timescale. For example, Policemanl's response included a number of temporally overlapping decisions and communications (e.g., the hospital designation and the traffic exclusion zone); it seems implausible that 
Policeman 1 might have learnt about coping with these temporal constraints. More generally then, it appears that tabletop exercises cannot provide training in the coordination of planning which is opportunistic and consequently fragmented (Section 1.1).

Training coordinated action. The table top exercise disclosed the dependencies between agents that may dictate the sequences of their planned actions. For example, Fireman1 and Railwayman2 appeared to create a mutual dependency wherein electrical isolation must be provided on request by the fire brigade who will need confirmation of the isolation before they can proceed with the rescue operation. In this way, tabletop exercises appear to train the recognition and negotiation of the dependencies between agents dictating sequences of actions, as necessary for coordination.

Yet the process of discussion did not, and could not simulate the visible execution of plans. The tabletop exercise was therefore unable to recreate the reactivity between actions of different agents. Consider the decision by Policeman1 to establish a traffic exclusion zone around St Michael's church without considering the vital question of access to the accident. Although Fireman2 and Ambulanceman1 later identified the need for access to the accident from the other side of the embankment, would they have earlier scaled the wrong side of the embankment? It would appear that tabletop exercises are unable to train the reactivity of actions that supports coordination.

Training coordinated communication. The tabletop exercise exposed all participants to the same information at the same time; it did not simulate the progressive dissemination of information that occurs during emergency operations. That information was generally authoritative (i.e., the Moderator's descriptions) and validated (i.e., the view of the model); it was also accurate, with the exception of Mrs Jones' initial report of a "bang on the railway." It would appear that 
tabletop exercises are unable to recreate the conditions of information dissemination required for training coordination.

Because the tabletop exercise did not simulate the 'channels of information' and their contents that occur during a real emergency operation, it could neither re-create the asynchrony of communications; all participants could address all others directly and immediately. More generally then, tabletop exercises appear unable to train the rapid passing-on of information necessary for coordination.

Training coordinated roles and knowledge. There was no evidence in the tabletop exercise of differences between the nominal and real divisions of labour, and for two apparent reasons. First, the scenario did not present the exigencies of a catastrophe which might force a difference between the nominal and real divisions of labour. Second, each trainee was playing the role of their entire agency, rather than the role of a single agent. Without evidence of the divisions of labour being configured, it may only be concluded that the tabletop exercise did not provide training in the negotiation of that configuration, as necessary for coordination.

However, if the real division of labour requires a partial redundancy in knowledge and skills, that redundancy could be learnt through the discussion of individual responses. For example, all the trainees appear to learn about the issues confronting the railwaymen in obtaining track isolation - and they learn that the wreckage will almost certainly have already short-circuited the current. Equally, the discussion would extend the cognitive horizon of each participant. More generally then, tabletop exercises appear to provide the redundant knowledge needed for coordinating the configuration of roles, for example, in recognising which agents need extra support.

In summary, the tabletop exercise appears to provide valuable, but incomplete training in coordination. Participants learn collectively to interpret contingency plans, to resolve conflicts between different priorities, and to recognise and negotiate mutual constraints. They may 
also extend their cognitive horizons, as necessary for mutual support and configuring the real division of labour. However, the exercise does not provide training in the reactivity of planning to events, or in the reactivity of actions to the actions of others. It can provide no training in planning and decision-making under high temporal constraints. Neither does the tabletop exercise appear to provide training in the dissemination of information, nor in the asynchrony of communications.

\section{Requirements for simulators for coordination training}

Given the agenda of coordination training needs, and the assessment of how well the tabletop exercise currently meets those needs, the following requirements can be recognised for future computer-based training simulations. The requirements are for simulations which retain the strengths of the tabletop exercises whilst making good its weaknesses.

Coordination issues emerge only with collective activity and therefore training simulators are required which re-create the collective activity of emergency management. However this requirement does not mean that training must in principle also be collective - in other words, that the entire management structure needs to be trained together. The functions and roles of some emergency managers may be simulated, either by the simulator itself or by trained assistants.

Training simulators are required which support learning how to plan emergency operations, complimenting the learning of contingency plans in the classroom. In particular, learning collectively to interpret contingency plans in a way that makes individual mis-intepretations apparent (n.b., a deficiency of the tabletop exercise) is required. Similarly, there is a requirement to maintain training in the recognition and resolution of conflicts. Implied is the requirement for a facility to collect a record of concurrent planning and plan revision activity; this record would be used in structured de-briefings following the training exercise to diagnose plan interpretation issues. 
Training simulators are required to support 'learning by experience' of the planning process, not least because plans made during emergency operations are dictated by the experience of the time available for planning. Learning by experience contrasts with the 'learning by discussion' of a tabletop exercise. It presumes the requirement for the simulator to provide a dynamic, 'event-driven' domain model. Here, a domain model is a presentation of both the catastrophe (perhaps in terms of structures, hazards and people) and the emergency operation in the field (perhaps in terms of resources, actions and action responses). The goals of the emergency management are to bring the domain model into some desirable state. A domain model which is dynamic and event-driven represents a catastrophe and emergency operation in terms of a time course of events (both predictable and random) and their consequences. By presenting its scenario through a real time domain model, a training simulator would stand in contrast with the tabletop exercise which presents its scenario through a spoken script and a static model.

Training simulators are required which support learning of the reactivity between different agents' actions. Since the reactivity of actions occurs through the visibility of actions and their effects, this requirement presumes that the domain model will be responsive, that is to say, that the time course of domain events changes in response to the instructions issued by the trainee emergency managers. Making the scenario responsive further contrasts with the scenario of the tabletop exercise which is not responsive.

Training simulators are required which support learning of the mutual dependencies between agents' actions. Since these dependencies are established by negotiation, the simulator is required to provide the channels of communication between agents through which negotiation can occur. Most simply, this requirement may imply a multi-user simulator with a common interface shared by the entire group of trainee emergency managers. Negotiation of dependencies would then be achieved by face-to face discussion, as for the tabletop exercise. 
Training simulators are required to support learning of coordinated communications, and therefore are required to re-create the channels of communication between agents during real emergency operations, and the restrictions in those channels. This requirement implies a multiuser simulator which separates the trainee emergency managers, each having their own interface to the simulator through which they might communicate with other agents and agencies.

Training simulators are required to support learning of the rapid dissemination of authoritative, validated and accurate information. This requirement presumes that the user interfaces to the training simulator will present trainee emergency managers with the kinds of information they would be obtaining at any given time during a real operation. Since different agents receive different information depending on their roles, locations and communication channels, the different interfaces to the simulator are required to be 'role specific', that is, to provide a view of the scenario (i.e., of the domain model) appropriate to the role of the trainee. Equally, learning to disseminate information rapidly requires learning about the asynchrony of communications between different agents. The simulator is therefore required to provide restricted channels of communication between the trainees, simulating the channels available during real emergency operations.

Training simulators are required which provide training in the negotiation of divisions of labour. The simulator so far described should promote such training where the tabletop exercise failed. First, the dynamic, event-driven domain model of the simulator would provide the exigencies of the emergency operation necessary to make specific divisions of labour an issue; second, the role-specific user interfaces of the simulator would require a trainee to enact their individual role as an agent, rather than the global role of their agency. The negotiation of labour divisions is underwritten by the redundancy of agents' task knowledge, currently supported by the discursive mode of learning of the tabletop exercise. Since the training simulator supposes a mode of learning 'by practising', the same process of 
discussion by which trainee emergency managers extend their cognitive horizons would need to be provided by debriefing sessions based on playbacks of the training task, allowing trainees to discuss their earlier behaviours with regard to the optimality of the labour division.

Meeting the Requirements and the State-of-the-Art in Emergency Management Training Simulators

In this paper requirements have been identified for training simulators which might improve coordination in the management of emergencies. The requirements were identified by assessing a typical tabletop exercise with regard to an agenda of coordination training needs. The requirements produced are abstract and for two reasons. First, the requirements are presumed at the current time to apply generally across a range of catastrophe types and across internationally different ways of organising emergency agencies. Second, computer-based simulations for training emergency management are presently in their genesis and so such high-level requirements may well be most appropriate. Consider the example of ICCARUS, a state-of-the-art simulator for training fire brigade officers in managing large scale fires involving up to fifteen fire tenders.

The ICCARUS simulator was commissioned by the Learning Technologies Unit of the Employment Department and is in current use with the UK fire brigade. A comprehensive report on the simulator, and the method of its development, can be found in Workhouse (1992). ICCARUS consists of a set of modules for creating and running different event-based scenarios, such as a fire in a cinema in use by the public. The simulator trains fire officers individually, and a typical training session runs as follows.

To begin, the simulator provides the trainee with a spoken introduction to the catastrophe scenario, explaining that they are currently driving towards a five pump (fire tender) fire. The simulator's video monitor now shows a sequence of photographic stills of a route 
though an urban area, during which can be heard various recorded radio messages concerning the incident and simulating the radio traffic being relayed to the fire control centre.

The scenario soon describes the trainee as entering the mobile Command Unit at the scene of the emergency operation. The trainee must now take over as Officer-in-Charge of the operation. They are briefed by the officer standing down and using the standard protocol: "What have I got? What messages have you sent? "What have you done?" The part of the officer standing down is played by the simulator; the dialogue is conducted by the trainee interrogating the officer through the simulator's WIMP interface, textual responses being displayed on the computer monitor. The monitor provides a strategist's representation of the emergency operation: a plan of the building and the current state of the fire; the current deployment of 'resources' and those available for deployment. This representation re-creates paperbased planning aids typically used in managing emergency operations. It is a view of the computational domain model of the emergency operation as that model is executed by the simulator's transputer.

The domain model is an event-driven, dynamic model simulating the progress of the fire through the building and the location and properties of objects such as hazardous materials; in addition, it simulates the location and behaviours of firemen and agents of other emergency agencies and of members of the public trapped in the building, and it models the location and behaviours of equipment such as pumps. In their role as Officer-in-Charge, the trainee is able to manage the emergency operation by intervening in the state of the domain model, for example, by requesting more pumps and officers, moving pumps and directing specialist teams, etc. These actions are taken by issuing commands, for example, to deploy a breathing apparatus team, the trainee uses the mouse to select the icon 'BA Deploy' and an entrance to the building. The domain model responds to these instructions, for example, the breathing apparatus team are able to act with semi-intelligence to enter the building, locate trapped 
people or fight the fire. So, the effects of the trainee's instructions, the progress of the fire, and the behaviours of up to 200 'automaton' firemen and other agents are each visible to the trainee.

Photographic stills and video film are stored on videodisc within the simulator. Video film of a fire at a disused cinema was recorded for the express purpose of the simulator's development. The simulator also provides (from CD-ROM) an acoustic environment of voices and noises typically heard at 'firegrounds'. As events unfold within the executing domain model, the simulator selects appropriate visual images and sounds, for example, distraught people stating that someone is trapped in the building, or other agencies demanding information. The intended effect is to bombard the trainee and induce them to suspend disbelief (see Hennessey and O'Shea, 1993). The training session finishes when the fire is controlled, or the building lost. All the scenario events and the trainee's actions are recorded and the record used to debrief the trainee. The debrief addresses at each point whether the trainee has an overview, whether they are deploying resources optimally, whether communications are optimal, and whether the trainee is being reactive to events.

ICCARUS is one of only very few simulators in existence for training emergency management. Developed in 1991, its continuing uptake by the end-user organisation confirms the system as being stateof-the-art. As apparent from its debriefing structure, ICCARUS is intended to provide comprehensive training in emergency management, including training in coordination issues.

With reference to the general requirements identified in the previous section, it is apparent that ICCARUS enables the trainee to learn how to plan by experience. It is able to do this because its domain model is dynamic, it represents the time course of events of the emergency operation, and it is responsive to the decisions taken by the trainee. The simulator also supports post-task review and debriefing by collecting a structured record. However the trainee does not learn to plan within a collective management structure. Although they instruct 
fire officers and provide information requested by the emergency management of other agencies, the trainee's decision-making is independent: their role is one of autocrat, not oligarch. Accordingly, the trainee is unable to learn collectively to coordinate planning, action, communication and task knowledge. In part, it is the very sophistication of the ICCARUS domain model which precludes the simulator being a multi-user system: it is likely that current computational techniques are unable to provide domain models which are dynamic, event-driven, which simulate intelligent behaviour - and which are responsive to the independent interventions of a number of trainees. There are examples of multi-user training simulators in related domains (for example, VISTRAIN in use with the Scottish Police College - see LTU, 1992), but these do not have computational domain models, and therefore do not meet the requirement for being responsive.

To conclude, consideration of ICCARUS indicates the degree to which coordination training is supported by current state-of-the-art emergency management simulators. The requirements identified in this paper are intended to better direct the development of simulators that can improve the coordination of emergency operations - and so also their effectiveness.

\section{References}

Borodzicz, E., Aragones, J.I., and Pidgeon N. (1993). Risk communication in crisis: meaning and culture in emergency response organisations. Proceedings of the 4th Conference on European Technology and Experience in Safety Analysis and Risk Management, Society of Risk Analysis (Europe), 19-20 October 1993, Rome. 
Cicourel, A.V. (1990). The integration of distributed knowledge in collaborative medical diagnosis. In Galegher J., Kraut R. and Egido C., (eds) Intellectual Teamwork. Hillsdale, NJ: Lawrence Erlbaum.

Dowell, J. (1995). Coordination in emergency operations and the tabletop training exercise. Le Travail Humain, vol. 50, pp. 85-102.

Emery, F.E. and Trist, E.L. (1973). Towards a Social Ecology. New York, Plenum.

Fennell, D. (1988). Investigation into the King's Cross Underground Fire. Department of Transport (HMSO): London.

Hennessey, S. and O'Shea, T. (1993). Learner perceptions of realism and magic in computer simulations. British Journal of Education Technology, 24 (2), 125-138.

Hidden, A. (1989). Investigation into the Clapham Junction Railway Accident. Department of Transport (HMSO): London.

Hutchins, E. (1990). The technology of team navigation. Galegher J., Kraut R. and Egido C., (eds) Intellectual Teamwork. Hillsdale, NJ: Lawrence Erlbaum.

Lagadec, P. (1990). States of Emergency: Technological Failures and Social Destablisation. London: Butterworth-Heinemann.

LTU (1992). Crowd control. Report of the development of the control of crowds at major spectator events. Report produced for and published by the Learning Technologies Unit, Employment Department: Sheffield.

Overy, B. (1993). The different types of exercise: when to use them. In Proceedings of Disaster '93, :5th Disaster Prevention and Limitation Conference (Volume 2), 16 September 1993. University of Bradford.

Rasmussen, J. Brehmer, B. and Leplat, J. (eds.) (1991). Distributed Decision Making: Cognitive Models for Cooperative Work. Chichester: Wiley and Sons.

Samurçay, R. and Rogalski, J. (1993). Cooperative work and decision making in emergency management. Le Travail Humain, 56 (1), 53 77 . 
Workhouse Ltd, (1992). ICCARUS: Intelligent command and control: acquisition review using simulation. Report produced for and published by the Learning Technologies Unit, Employment Department: Sheffield.

\section{Author's Note}

This work was supported by the Environment program of the European Community under the MUSTER project (Multi-User Simulation for Training Emergency Responses). I am grateful to the Learning Technologies Unit at the Employment Department, Sheffield for access to ICCARUS. The conclusions in this paper are entirely those of the author and should not be assumed to represent the views of the organisations mentioned. 
